\title{
Linfoma Primário de Tireoide Mimetizando Carcinoma Anaplásico
}

\section{Primary Thyroid Lymphoma Mimicking Anaplastic Carcinoma}

Ana Luiza Viana Pequeno ${ }^{1}\left(\mathbb{C}\right.$, Jonatas Catunda de Freitas ${ }^{2}\left(\mathbb{D}\right.$, Ana Carla Albuquerque dos Santos ${ }^{2} \mathbb{D}$, Priscilla Mariana Freitas Aguiar $^{3}\left(\mathbb{D}\right.$, Matheus Augusto Mesquita Fernandes ${ }^{1} \mathbb{D}$, Wellinton Alves Filho ${ }^{4}$

1. Discente do curso de Medicina pela Universidade Federal do Ceará (UFC). 2. Residente de Cirurgia Cabeça e Pescoço da Universidade Federal do Ceará (UFC). Fortaleza, CE, Brasil. 3. Residente de Patologia do Hospital Universitário Walter Cantídeo (HUWC), Fortaleza, CE, Brasil. 4. Docente do Departamento de Cirurgia da Universidade Federal do Ceará (UFC).

\section{Resumo}

\begin{abstract}
Introdução: o linfoma primário de tireoide é uma neoplasia rara, agressiva, cuja manifestação pode assemelhar-se ao carcinoma anaplásico. Relato do caso: descreveu-se o caso de uma paciente de 59 anos com crescimento de massa cervical anterior volumosa em um mês associada à disfonia. Tomografia computadorizada revelou aumento da tireoide com traqueia desviada e linfonodomegalia. Histopatológico confirmou linfoma difuso de grandes células $\mathrm{B}$ de alto grau. Paciente foi, celeremente, submetida à terapia e obteve boa resposta. Conclusão: em pacientes com rápido crescimento de massa em topografia tireóidea, a hipótese de linfoma deve ser elencada, sendo primordial diagnóstico precoce e conduta agressiva.
\end{abstract}

Palavras-chave: Tireoide. Cirurgia. Cabeça e pescoço. Linfoma.

\begin{abstract}
Introduction: primary thyroid lymphoma is a rare, aggressive neoplasm, whose manifestation may resemble anaplastic carcinoma. Case report: a 59-yearold patient with an increasing anterior cervical mass in one month, associated with dysphonia. Computed tomography revealed enlargement of the thyroid with tracheal deviation and lymph node enlargement. Histopathology confirmed diffuse high-grade large B-cell lymphoma. The patient was promptly submitted to therapy and obtained a good response. Conclusion: in patients with sudden mass growth in thyroid topography, the hypothesis of lymphoma should be considered, with rapid diagnosis and aggressive conduct being essential.
\end{abstract}

Keywords: Thyroid. Surgery. Head And Neck. Lymphoma.

\section{INTRODUÇÃO}

A glândula tireóidea é comumente alvo de doenças neoplásicas'; entre as quais está o linfoma primário de tireoide (LPT), subtipo de linfoma não hodkin que representa de 1 a $5 \%$ dessas malignidades 2 e de 1 a $2 \%$ dos linfomas extranodais ${ }^{1},{ }^{2}$. Essa rara condição, por definição, envolve a tireoide e pode ou não estar acometendo os linfonodos locais no momento do diagnóstico, mas deve ser excluída das hipóteses diagnósticas se estiver além desses limites ${ }^{2}$. Sua apresentação clínica costuma ser como uma massa indolor de rápido crescimento no compartimento cervical anterior, sem sintomas constitucionais, mas compressivos ou infiltrativos ${ }^{2}$. Em alguns casos, o LPT mimetiza o carcinoma anaplásico, necessitando diferenciação microscópica ${ }^{3}$. 0 diagnóstico precoce de linfoma tem substancial relevância para o seu melhor manejo ${ }^{2}$, o qual vai variar de acordo com o histopatológico e o estágio do tumor ${ }^{4}$. Neste artigo, relatou-se o caso de uma paciente com LPT que teve resposta excelente ao tratamento.

\section{RELATO DE CASO}

Mulher de 58 anos procurou atendimento médico devido ao aparecimento de massa cervical volumosa em topografia da tireoide de rápido crescimento em um mês associado à rouquidão. Negava dispneia, perda de peso considerável nos últimos meses ou sudorese. Ao exame físico, a tireoide apresentava-se, difusamente, aumentada e com consistência endurecida. Tomografia cervical evidenciou aumento das dimensões da tireoide, principalmente no lobo esquerdo, comprimindo e desviando a traqueia para a direita, e linfonodomegalia cervical de aspecto ovalada na cadeia da jugular interna, com 1,3 cm no maior diâmetro, e na cadeia espinhal acessória esquerda, com 2,0 cm.

Devido à forte suspeita inicial de carcinoma anaplásico da tireoide, decidiu-se realizar traqueostomia transtumoral para garantir a via aérea e ressecção de todo o componente anterior à traqueia da lesão para estudo histopatológico. O resultado da biópsia mostrou linfoma difuso de grandes células $B$ de alto grau, confirmado pela imuno-histoquímica. No estadiamento, a paciente apresentou múltiplos nódulos pulmonares e múltiplas linfonodomegalias retroperitoneais e mesentéricas. Iniciou tratamento com seis sessões de quimioterapia seguindo o protocolo R-CHOP com rituximabe, $375 \mathrm{mg} / \mathrm{m}^{2} \mathrm{EV}$, no D1, ciclofosfamida, $750 \mathrm{mg} / \mathrm{m}^{2} \mathrm{EV}$, no D1, doxorrubicina, $50 \mathrm{mg} / \mathrm{m}^{2}$

Correspondente: Ana Luiza Viana Pequeno. Endereço: Rua Alexandre Baraúna, 949, Rodolfo Teófilo, Fortaleza-Ce, CEP: 60.430-160. E-mail: analuizapequeno@hotmail.com

Recebido em: 4 Jan 2019; Revisado em: 6 Fev. 2020; Aceito em: 12 Fev. 2020 
$\mathrm{EV}$, no D1, vincristina, $1,4 \mathrm{mg} / \mathrm{m}^{2} \mathrm{EV}$, no $\mathrm{D} 1$, e predinisona, 100 $\mathrm{mg}$ VO, do D1 ao D6, a cada três semanas. A paciente concluiu o tratamento e teve resposta completa. Encontra-se bem, sem queixas e sem sinais de recidiva, 12 meses após o término do tratamento.

Figura 1. Imagens coradas pelo HE vistas à microscopia mostram proliferação linfoide atípica composta por linfócitos de tamanhos variados, com nucléolos evidentes e discreto pleomorfismo nuclear. Há áreas de fibrose e destruição folicular.
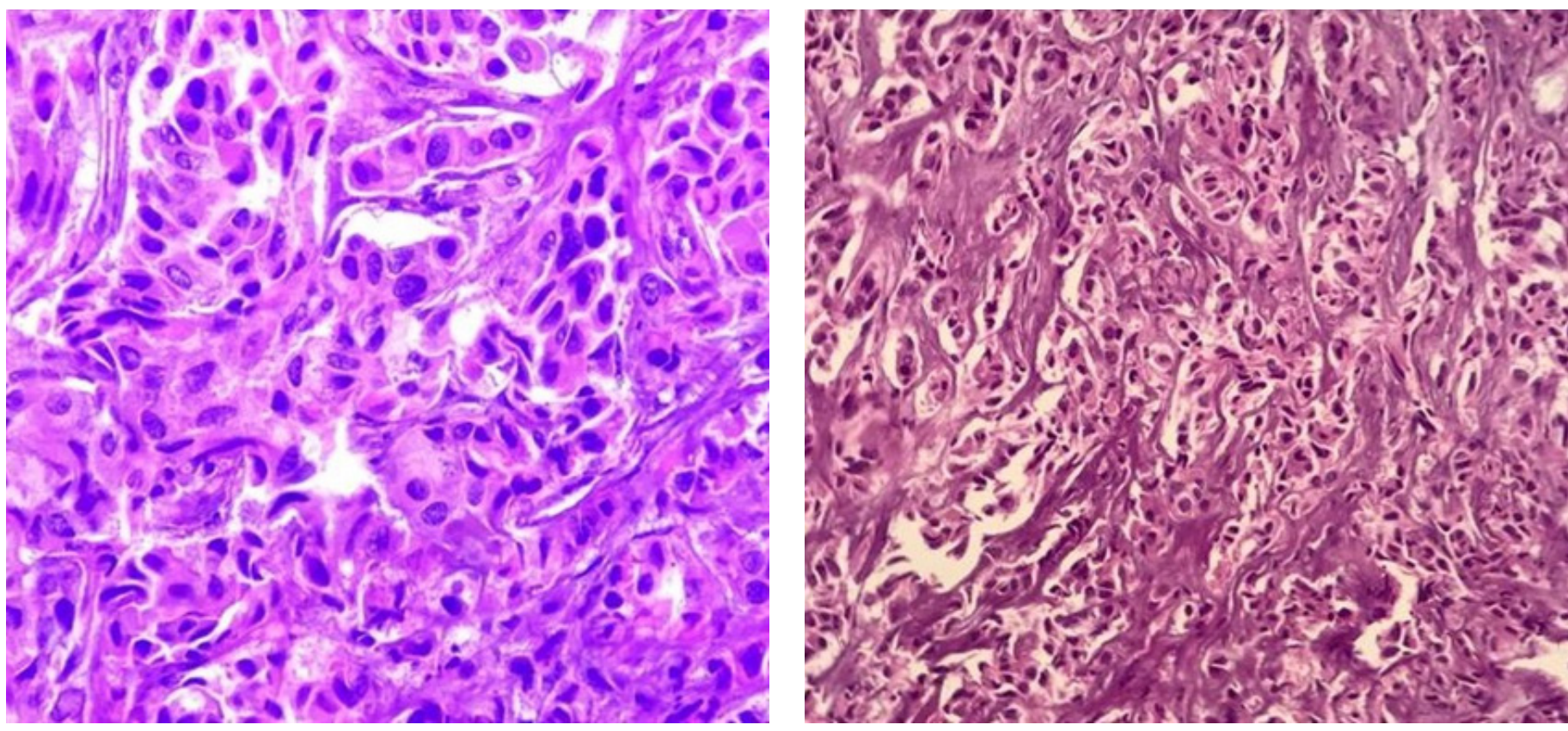

Figura 2. Topografia cervical antes e depois da ressecção.
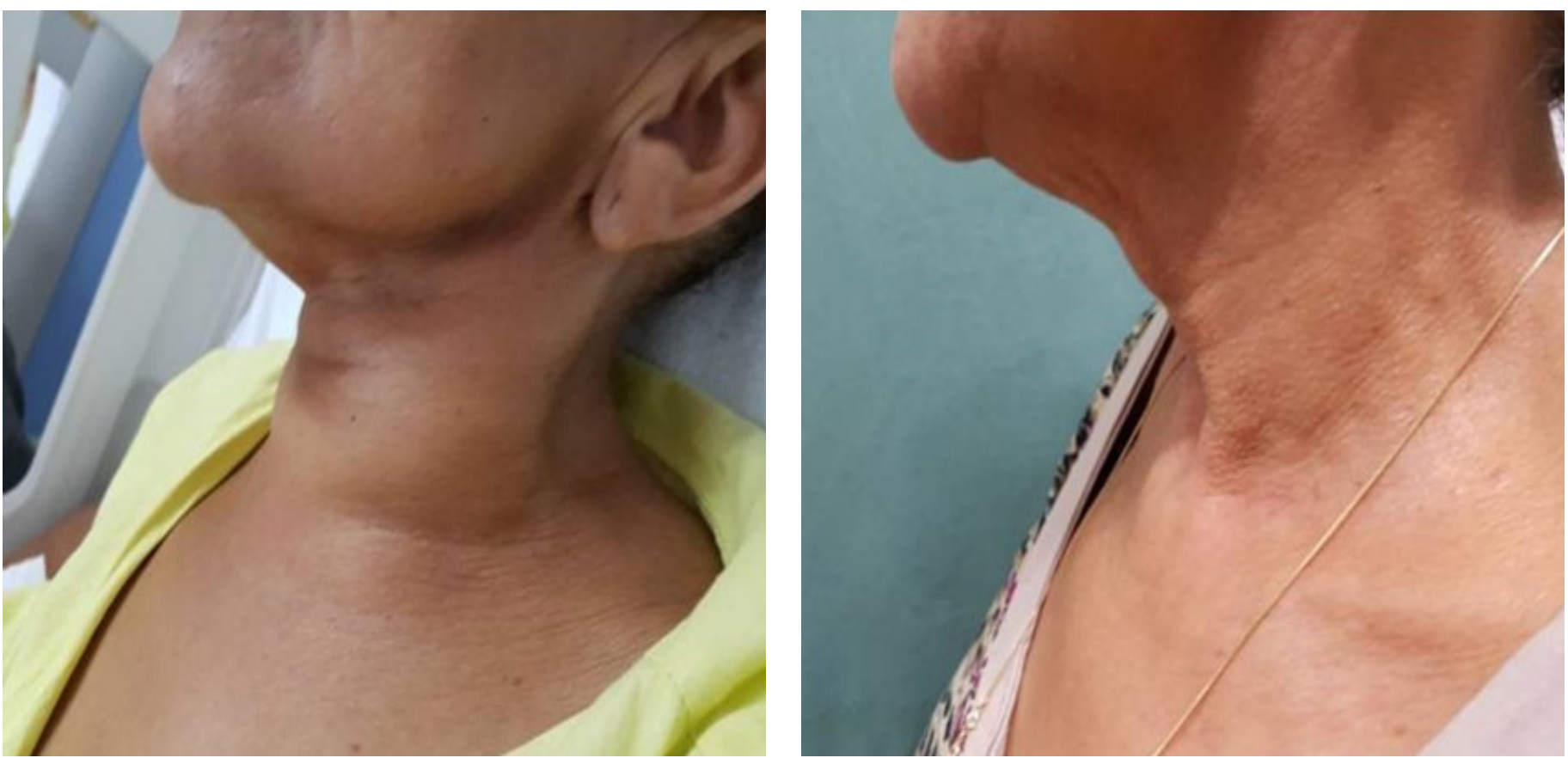
Figura 3. Intraoperatório da traqueostomia transtumoral.

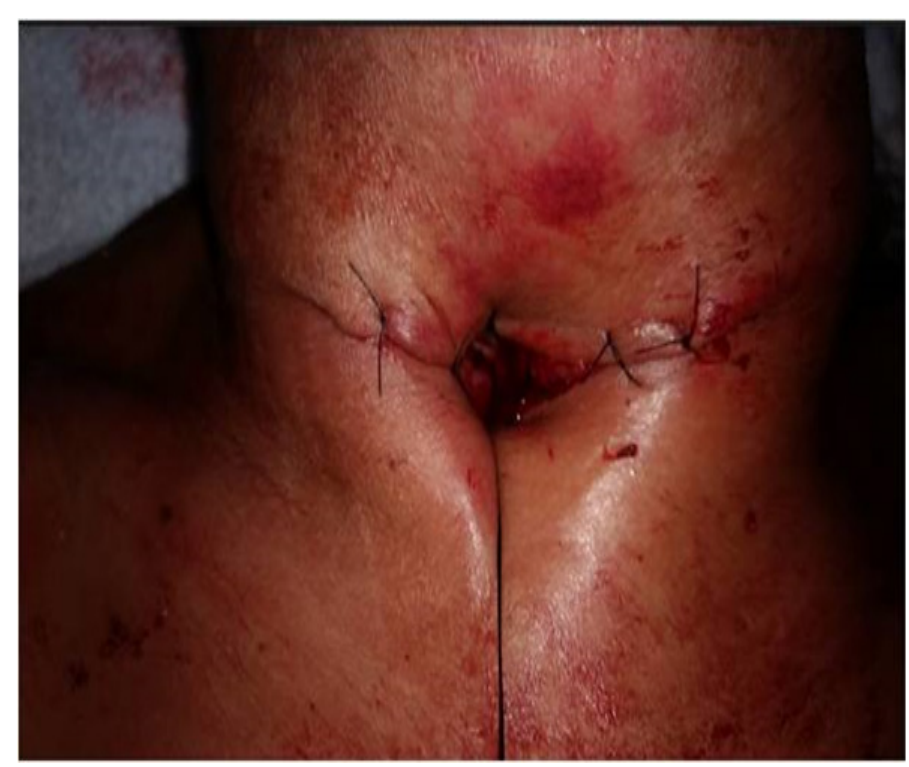

\section{DISCUSSÃO}

Linfomas de tireoide são neoplasias malignas raras, tendo uma incidência anual de cerca de 2 por 1 milhão de indivíduos ${ }^{4}$. São classificados em primários, quando crescem a partir da própria glândula tireóidea, e em secundários, quando provenientes de metástases linfonodais a distância, uma condição disseminada comumente atrelada ao pior prognóstico ${ }^{5}$. Há, ainda, diferenças clínicas e epidemiológicas. A incidência de metástase tireoidiana em pacientes com linfoma varia de 11 a $27 \%$, mas muitos são apenas achados em necropsias devido a raras manifestações clínicas como o bócio, presente em menos de $0,06 \%$ dos acometidos ${ }^{1,3}$. Ressalte-se que a intrínseca relação entre tireoidite de Hashimoto e linfoma primário não é observada nos tumores secundários ${ }^{1}$, explicando-se isso com base nas alterações genéticas que são propiciadas pela doença autoimune ${ }^{1}$. Essa diferenciação de linfomas de tireoide entre primário e secundário é determinante para que a terapêutica de ambas as situações sejam adequadas ${ }^{5}$. Enquanto tiroidectomias costumam ser uma alternativa para os casos primários, em secundários, cirurgias extensivas não são indicadas e quimioterapias preferidas 5 .

O LPT costuma ocorrer na sétima década de vida e acomete mais mulheres que homens na proporção $8: 1^{4}$. Pacientes com tireoidite de Hashimoto apresentam risco relativo de desenvolver LPT de 67 vezes maior se comparada à população em geral ${ }^{4}$. Entretanto, apenas $0,5 \%$ de todos os casos de doença de Hashimoto desenvolvem tal linfoma².

Quanto à apresentação clínica, pode ser na forma de bócio ou nódulos discretos. Dois terços dos pacientes são assintomáticos, e os demais apresentam sintomas resultantes da compressão de estruturas cervicais, como a faringe e laringe, desencadeando dispneia, estridor e tosse; esôfago, levando à disfagia; vasos sanguíneos, apresentando-se como hemoptise ${ }^{1}$. Febre, perda de peso e suores noturnos, descritos como sintomas $B$, aparecem em cerca de $10 \%$ dos casos ${ }^{1}$. No tocante à função tireoidiana, 90\% dos pacientes sem acompanhamento endócrino prévio encontram-se eutireoidianos, enquanto o hipotireoidismo está presente nos demais ${ }^{1}$. A dor é relatada em $12 \%$ dos pacientes ${ }^{4}$. Nesse contexto, a duração média dos sintomas até o diagnóstico é de quatro semanas ${ }^{2}$. $\mathrm{O}$ acometimento respiratório e a redução do retorno venoso por causa da síndrome da veia cava superior implicam tratamento imediato 4 .

Após a suspeita clínica, o próximo passo para o diagnóstico é a ultrassonografia ${ }^{3}$. Esse método de imagem sugere LPT ao identificar uma lesão assimétrica marcadamente hipoecogênica com padrões lineares ecogênicos, sendo necessária a biópsia para confirmação ${ }^{1}{ }^{3}$. A aspiração com agulha fina acompanhada por citologia e imuno-histoquímica tem alta sensibilidade e especificidade para o diagnóstico ${ }^{1}$. Quando o resultado for inconclusivo, o seguimento consiste em biópsia por agulha grossa -core biopsy- ou biópsia cirúrgica aberta ${ }^{5}$.

O LPT pode ser subdivido histopatologicamente em linfoma de células $T$, plasmocitoma e linfoma de células $B$, sendo este último a maioria dos $\operatorname{casos}^{5}$. O linfoma de células B pode ainda ser classificado como Hodgkin ou não Hodgkin, o qual compreende as variantes: linfoma de tecido linfoide associado à mucosa (MALT); linfoma difuso de grandes células B (LDGCB) e variante mista ${ }^{5}$. O linfoma MALT, responsável por $20-30 \%$ dos casos, costuma ter crescimento indolente 5 . Já o LDGCB, 70-80\% dos LPT, é mais agressivo ${ }^{5}$. A variante mista apresenta comportamento mais similar ao LDGBC5. Os achados compatíveis com LDGCB incluem falta de coesão celular, pleomorfismo com nucléolo proeminente, várias figuras mitóticas presença de corpos linfoglandulares ${ }^{5}$.

A paciente estudada, apesar de não apresentar dispneia ou demais sintomas respiratórios, apresentava estenose de traqueia visualizada na TC. Para garantir vias aéreas pérvias, optou-se por fazer traqueostomia transtumoral. Nesse dia, foi realizada uma biópsia que revelou massa cervical bastante sugestiva de linfoma de tireoide. Os outros exames foram realizados devido à necessidade de descartar diagnósticos diferenciais, observando-se nos resultados da TC a presença de múltiplos nódulos pulmonares, que indica também possíveis implantes secundários e múltiplas linfonodomegalias retroperitoneais e mesentéricas, o que sugere doença proliferativa.

Por apresentar um curto espaço temporal para tamanho crescimento de massa cervical, o principal diagnóstico diferencial da nossa paciente era câncer anaplásico. Além de apresentar rápida evolução, esse tipo de neoplasia também predomina em pessoas do sexo feminino e em idosos, acima de 60 anos $^{3}$. O câncer anaplásico é o mais letal das malignidades da tireoide, com sobrevida média de seis meses ${ }^{3}$. Portanto, tem alta relevância a diferenciação histológica das duas neoplasias, que se baseia na presença de inclusões celulares e ausência de corpos linfoglandulares para caracterizar o câncer anaplásico5. Tais achados não corresponderam aos de nossa paciente, 
possibilitando diferenciação e caracterização como LPT.

O tratamento ainda é algo muito debatido, pois se tratando de uma condição rara, os estudos baseados em tal pauta ainda se mostram escassos ${ }^{4}$. A tendência atual é que se faça uma abordagem combinada ${ }^{2,5}$. Em razão da alta recidiva e das baixas taxas de sobrevivência, tireoidectomias totais não são mais usadas em larga escala ${ }^{4}$. Restringem-se aos casos de linfoma MALT limitado à glândula tireóidea ${ }^{4}$. O plano terapêutico mais utilizado é a associação de radioterapia com quimioterapia, aos quais os linfomas de tireoide são bastante sensíveis ${ }^{4}$. Amiúde cirurgias paliativas são necessárias para aliviar os sintomas que interferem na qualidade de vida do paciente ${ }^{5}$.

\section{CONCLUSÃO}

LPT é uma neoplasia rara que acomete, principalmente, mulheres com mais de 60 anos. Seu fator de risco mais relacionado é a tireoidite de Hashimoto, em face dos fatores genéticos associados. O diagnóstico deve ser suspeito em pacientes com massas cervicais anteriores, de rápido crescimento, que podem ou não estar associadas a sintomas compressivos, como dispneia, disfagia, rouquidão e hematêmese. Sintomas B e dor são pouco frequentes.

Ultrassonografia cervical é indicada nesses pacientes para avaliação inicial, mas apenas a biópsia apresenta confirmação diagnóstica. $O$ estudo citológico proveniente permite classificar o tipo de LPT, sendo o LDGBC a variante mais frequente. A terapia de escolha vai depender da extensão da lesão, mas a tendência é que seja combinada com quimioterapia e radiocirurgia. Atualmente, a cirurgia é indicada para casos estritos. Apesar de tratar-se de uma paciente com doença avançada, apresentou resposta completa ao tratamento.

\section{REFERÊNCIAS}

1. Mancuso S, Carlisi M, Napolitano M, Siragusa S. Lymphomas and thyroid: Bridging the gap. Hematol Oncology. 2018 Feb; 1-6. doi: https://doi. org/10.1002/hon.2504.

2. Pavlidis ET, Pavlidis TE. A Review of Primary Thyroid Lymphoma: Molecular Factors, Diagnosis and Management. J. Invest. Surgery. 2017 Oct. doi: http:// dx.doi.org/10.1080/08941939.2017.1383536.

3. Gu LS, Cui NY, Wang Y, Che SN, Zou SM, He W, et al. Comparison of sonographic characteristics of primary thyroid lymphoma and anaplastic thyroid carcinoma.
J Thorac Dis. 2017 Sep; 9(11): 4774-4784. doi: http://dx.doi.org/10.21037/ jtd.2017.09.48.

4. Stein SA, Wartofdky L. Primary Thyroid Lymphoma: A Clinical Review. Journal of Clinical Endocrinology and Metabolism. 2013 Aug; 98(8): 3131-3138. doi: https://doi.org/10.1210/jc.2013-1428.

5. Spielman DB, Badhey A, Kadakia S, Inman JC, Ducic Y. Rare Thyroid Malignancies: an Overview for the Oncologist. Clin Oncol (R Coll Radiol). 2017 Jan. 29(5): 298-306. doi: http://dx.doi.org/10.1016/j.clon.2017.01.041.

Como citar este artigo/How to cite this article:

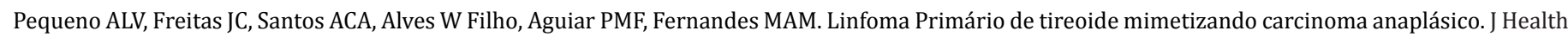
Biol Sci. 2020 J; 8(1):1-4. 